Rev. Chil. Pediatr. 57(6): 569-570, 1986

\title{
Síndrome de Rud
}

Dra. Isabel Avendaño B, ${ }^{1}$; Dr. Manuel Aspillaga H. ${ }^{2}$

\section{Rud syndrome}

The diagnosis of Rud syndrome was done in a 2.7/12 year old male with ichthyosis, hipogonadism and mental retardation. The outstanding clinical features of this syndrome and its autosomic recessive mode of inheritance are emphasized.

(Key words: Ichthyosis, mental retardation, hipogonadism, autosomic, recessive inheritance, Rud syndrome).

Las jctiosis constituyen un conjunto de enfermedades que presentan alteración en la queratiniżación, caracterizada por descamación visible en diferentes sitios. Los síndromes con ictiosis son clinicamente diferenciables entre si por sus carac. teristicas hereditarias, manifestaciones $y$ defectos asociados e histología.

\section{Caso Clínico}

Varón, consultó por primera vez a los 15 días de edad, por presentar micropene y criptorquídea bilateral: padres jóvenes sanos, dos hermanas menores sanas, sin antecedentes familiares. Em. barazo deseado, controlado y sin incidentes. Nació mediante cesárea por hipertensión arterial materna. Pesó $3.200 \mathrm{~g}$, midió $51 \mathrm{~cm}$ de talla y su examen de recién nacido fue normal. Al mes de edad la cromatina $X$ resultó normal y el estudio cromosómico de linfocitos, con técnica de bandeo $\mathrm{G}$, dió como resultado 46,XY. A los 6 meses de edad fue sometido a exploración quirúrgica de los genitales internos, encontrándose testiculos en ambos lados: el del lado derecho estaba en el conducto inguinal, su conducto deferente y vasos sanguíneos eran normales, el epidídimo tenía un

1. Unidad de genètica, Hospital Luis Calvo Mackenna.

2. Profesor de pediatría, Facultad de Medicina, Universidad de Chile. meso largo y la via espermática era continua; el del lado izquierdo estaba en la cavidad abdominal junto con sus vias. El estudio histológico con. firmó que habia tejido testicular en ambos. El análisis cromosómico realizado de la biopsia gonadal también dió como resultado $46, X Y$. Fue controlado con prueba de hormona liberadora de gonadotropina $(\mathrm{GnRH})$ endovenosa, en la que no se detectó lúteotrofina (LH) plasmática, en el momento basal y una hora después de la inyec. ción; y la concentración de hormona folículo estimulante (FSH) plasmática fue de $0,3 \mathrm{~m}$ UI $x$ $\mathrm{ml}$ basal y $1,7 \mathrm{~m}$ UI $\mathrm{x} \mathrm{ml}$ una hora después. La glicemia y las reacciones serológicas para toxo. plasma, rubeóla, citomegalovirus y herpes simple (TORCH) fueron normales, como también la clectromiografia, las radiografias de cráneo y silla turca y el estudio funcionai de tiroides.

Se realizó un tratamiento con $5.000 \mathrm{u}$ de gonadotrofinas coriónicas (GNC), en dos oportunidades, sin resultados sobre el tamaño peneano. A la edad de 2 años y 7 meses su talla era $92,5 \mathrm{~cm}$, y la circunferencia craneana medía $41 \mathrm{~cm}$, retardo psicomotor; hipotonía ge neraliza. da; ictiosis en el abdomen y las superficies de flexión de las extremidades superiores; cara y cráneo normales; tórax simétrico; escroto pequeño, rugoso, poco pigmentado y vacio; pene muy pequeño de $1,6 \mathrm{~cm}$ de largo desde el pubis al extreno distal, de $0,6 \mathrm{~cm}$ de diámetro en el glande $y$ de $0,7 \mathrm{~cm}$ en el cuerpo; testículos 
palpables con dificuttad en los conductos inguj. nales. El diagnóstico de síndrome de Rud se hizo sobre la base de la presencia de jctiosis, oligofrenia e hipogenitalismo, faltando la evidencia de epilepsia que puede aparecer posterjormentc (Fig. 1 y 2).

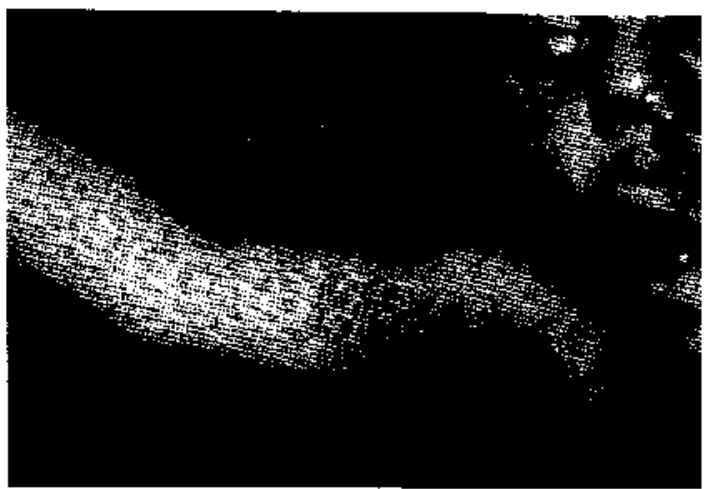

Figura 1. Ictiosis en cara de flexión de extremidades superiores.

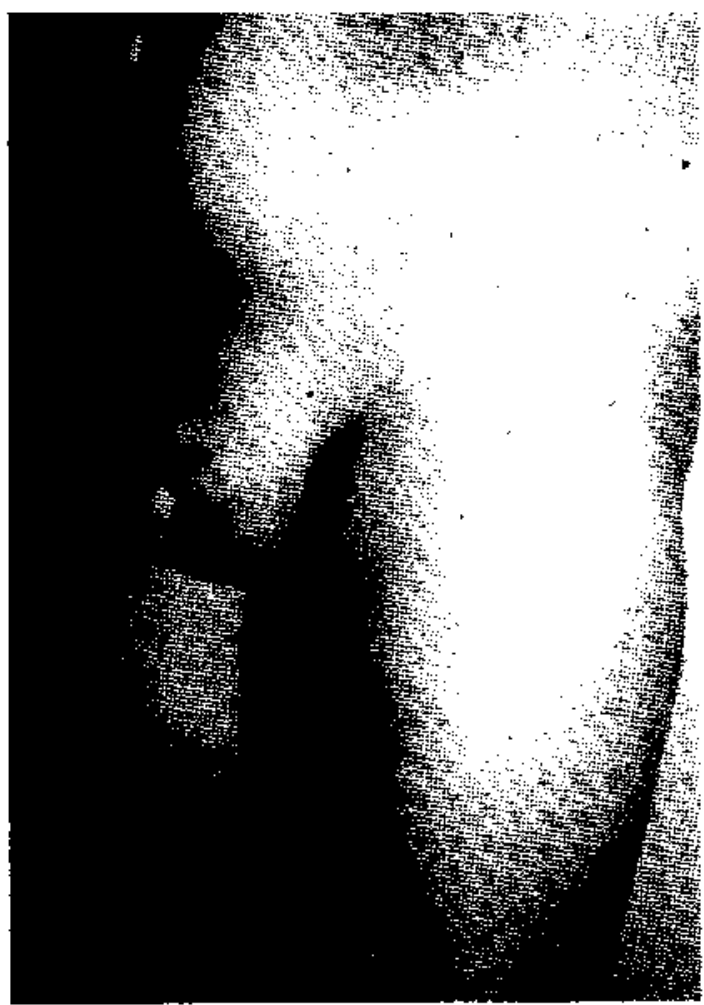

Figura 2, Genitales, micropene.

\section{COMENTARIO}

En 1927 Rud descubrió un sindrome caracterizado por epilepsia, infantilismo sexual, anemia macrocítica, polineuritis e ictiosis. Posteriormen- te se describió que además ten ian oligofrenia $y$ otros autores sugirieron que la ictiosis y el infantilismo son esenciales para el diagnóstico, y que la oligofrenia y epilepsia pueden no estar presen$\operatorname{tes}^{1,2,3,4}$.

Dentro de las afecciones con ictiosis asociadas e enfermedades neurológicas ${ }^{2.3}$ es necesario diferenciar las siguientes:

Síndrome de Sjörgren-Larson caracterizado por parálisis espástica, defíciencia mental, epilepsia, ictiosis laminar, cambjos semejantes a la retinitis pigmentosa, con herencia autosómica recesiva. E1 sindrome de Conradi se caracteriza por: ictiosis, deficiencia mental y herencia autosómica dominante. El síndrome de Refsun es una polineuropatía hipertrófica, con retinitis pigmentosa, sordera neural, ataxia cerebelosa e ictiosis leve, cuyas manifestaciones clinicas se hacen evidentes entre la primera y segunda década de la vida: cuya herencia es autosómica recesiva. La ictiosis ligada al cromosoma $X$ se acompaña también de deficiencia mental $y$ epilepsia. El síndrome de Rud, ya descrito, tiene herencia autosómica recesiva.

En nuestro caso se aconsejô a los padres limitar la familia, pues existen $25 \%$ de probabilidades de que el trastorno repita y, a su vez, las hermanas sanas pueden ser portadoras, instruyéndose sabre esta posibilidad a los padres. E] diagnóstico prenatal aún no es posible ya que las lesiones dérmicas se visualizan la mayoría de las veces en el lactante mayor.

\section{RESUMEN}

El diagnóstico de sindrome de Rud, puede basarse en la presencia de 3 signos clínicos básicos: ictiosis, que puede ser evidente desde ef primer arjo de vida, hipogonadismo y atraso psicomotor. El síndrome puede acompañarse de otros signos neurológicos como epilepsia, que puede estar presente desde el primer año de vida, o aparecer más tardíamente.

\section{REFERENCIAS}

1. Brith Defects Compendium. Daniel Bergstma 2nd. Edition, 1979, National Foundation Alan Liss, N.Y. pp. 800 .

2. Maldonodo, L.R. ef al: Neuroichthyosis with hipogonadisn (Rud syndrome). Int. J. Dermatol. 14: $347,1975$.

3. Textbook of Pediatrics. Waldo Nelson. 11 th Edition. 1979, Saunders Company, Phitadelphia, pp. 1899.

4. Wells, R.S., Kerr. M.: Genetic classification of ichthyosis. Arch. Dermatol. 92: 1, 1965. 